\title{
MoBAN: A Configurable Mobility Model for Wireless Body Area Networks*
}

\author{
Majid Nabi ${ }^{1}$, Marc Geilen ${ }^{1}$, Twan Basten ${ }^{1,2}$ \\ ${ }^{1}$ Department of Electrical Engineering, Eindhoven University of Technology, the Netherlands \\ ${ }^{2}$ Embedded Systems Institute, Eindhoven, the Netherlands \\ \{m.nabi,m.c.w.geilen,a.a.basten\}@tue.nl
}

\begin{abstract}
A good mobility model is an essential prerequisite for performance evaluation of protocols for wireless networks with node mobility. Sensor nodes in a Wireless Body Area Network (WBAN) exhibit high mobility. The WBAN topology may completely change because of posture changes and movement even within a certain type of posture. The WBAN also moves as a whole in an ambient network. Therefore, an appropriate mobility model is of great importance for performance evaluation. This paper presents a comprehensive configurable mobility model MoBAN for evaluating intraand extra-WBAN communication. It implements different postures as well as individual node mobility within a particular posture. The model can be adapted to a broad range of applications for WBANs. The model is made available through $h t t p: / / w w w . e s . e l e . t u e . n l / n e s /$, as an add-on to the mobility framework of the $\mathrm{OMNeT}++$ simulator. Two case studies illustrate the use of the mobility model for performance evaluation of network protocols.
\end{abstract}

\section{Categories and Subject Descriptors}

C.4 [Performance of Systems]: Modeling Techniques; Performance Attributes; I.6 [Simulation and Modeling]: Model Development

\section{General Terms}

Algorithms, Performance, Theory.

\section{Keywords}

Mobility model, Wireless body area network, Performance evaluation, Network simulator, OMNeT++, MiXiM framework.

\footnotetext{
*This work was supported by the Dutch innovation program Permission to make digital or hard copies of all or part of this work for personal or classroom use is granted without fee provided that copies are not made or distributed for profit or commercial advantage and that copies bear this notice and the full citation on the first page. To copy otherwise, to republish, to post on servers or to redistribute to lists, requires prior specific permission and/or a fee.

SIMUTOOLS 2011, March 21-25, Barcelona, Spain

Copyright (c) 2011 ICST 978-1-936968-00-8

DOI 10.4108/icst.simutools.2011.245511
}

\section{INTRODUCTION}

In the last decade, an increasing interest has been observed for wireless sensor network (WSN) technology in human body related applications. Several sensor devices may be deployed on or in a human body to sense the vital biological signals of the body. These sensors then communicate together forming a Body Area Network (BAN). Wireless technology makes the communication much easier and more comfortable for humans, thus enabling more applications for such networks (WBAN).

There are several interesting application areas of WBANs such as health care and sports. Continuous monitoring of elderly people or patients, for example, is really in demand as these groups are increasing in number. WBANs together with an ambient network form a secure environment for elderly people and patients to support them in their daily life, while being monitored by care workers.

Body area networks differ from typical large-scale wireless sensor networks in many aspects. Biosensor devices have to meet special constraints, such as a very small size, light weight, ultra-low power consumption, and tight performance requirements of the running application. Moreover, the characteristics of the wireless channel are different and links are in general low quality and time-variant. Thus, the proper architectures and protocols for wireless communication in these networks are still under active research. The research is being conducted on various aspects of different network layers (e.g. physical layer, medium access control, and link layer). Performance evaluation of the proposed protocols is an important phase of such research. Performance evaluation is typically done using simulations or real experiments. Although real experiments provide more reliable and precise results, simulation is still of great importance because it is less expensive, extensible to larger scale networks, and can be done in a shorter time. Simulation is also important because we can better observe the behavior of the protocol and detect potential bugs in the design and implementation process.

Mobility models have a big impact on the accuracy of simulations for wireless ad hoc and sensor networks with mobility in the network. Mobility models try to mimic the behavior of mobile nodes in reality by characterizing stochastic patterns of node movement. The right mobility model strongly depends on the application scenario. Since the sensors are deployed on the body in a WBAN, there is high node mobility and the network topology varies frequently. 
The channel quality and connection between nodes strongly depends on the relative position of sensor nodes. It makes the accuracy of the mobility model for WBANs more critical. In fact, simulating a protocol for WBANs without utilizing an appropriate mobility model is not reliable at all. There is individual mobility of sensor nodes deployed on different positions of the body as well as global movement of the whole body in the surrounding environment. A mobility model should capture all these aspects. Furthermore, it should provide sufficient flexibility for researchers to adapt it to their specific application scenarios within the WBAN domain.

This paper presents MoBAN (Mobility Model for BANs), a comprehensive and configurable mobility model for simulating wireless body area networks. The model is useful for simulating both intra- and extra-WBAN protocols. The former considers the communication between the sensor nodes within a WBAN on a body. Extra-WBAN protocols take care of communications between a WBAN and its environment, with potentially several body area networks as well as an ambient network. The model is configurable, which makes it usable for a large variety of applications.

There are several open source frameworks well structured for simulating protocols for WSNs and WBANs. For instance, the MiXiM framework [13] on top of the OMNeT ++ [2] discrete event simulator has a powerful library for simulating such kinds of wireless networks. The mobility framework of OMNeT++ makes it further suitable for mobile network simulation. Castalia [1],[19] is another framework on top of OMNeT ++ which is more specific for simulating WBAN protocols. It has an advanced channel model based on empirically measured data for the human body as the propagation medium as well as a radio model based on real radios for low-power communication. There are also several frameworks on top of other network simulators like NS-2/3 [4]. However, there is no freely available WBAN mobility model for simulating WBANs in any of these simulation frameworks. We have implemented our mobility model as an add-on to the mobility framework of the OMNeT++ network simulator so that it can be used for research on WBAN protocols.

The rest of the paper is organized as follows. The next section presents a survey on mobility models for wireless ad hoc and sensor networks existing in the literature. Our mobility model MoBAN is presented in Section 3. Section 4 describes implementation issues of MoBAN. Two case studies are discussed in Section 5, showing the need for a mobility model and the usability of MoBAN for performance evaluation of different communication protocols. Section 6 concludes.

\section{MOBILITY MODELS REVIEW}

Several mobility models have been presented in the literature of wireless ad hoc and sensor networks. A detailed survey of early proposed models can be found in [6]. This section firstly classifies mobility models and then briefly reviews some existing models of each class. Since there are many application-specific mobility models in the literature, we just review general and commonly used models. Finally, the only existing mobility model for wireless body sensor nodes is reviewed.
In general, we can classify mobility models into two major classes, namely singular node and group mobility models. In the former class, there is no correlation between the movement of different nodes and it models individual node mobility patterns regardless of the mobility of the other nodes in the network. The latter one takes a group of nodes into consideration which potentially have a particular relationship, which introduces correlation between their positions. Social activities of human beings are an example of group mobility for ad-hoc wireless mobile devices.

\subsection{Singular Node Mobility Models}

The Random Walk Mobility Model (RWMM) [27] is a commonly used singular node mobility model in which a node randomly selects a direction and a velocity value from a given range. The node then moves either with a constant time interval or until a constant distance is traveled. The movement takes place within a given rectangular space which is the simulation area. The node then repeats the random selection and movement process.

The Random Waypoint Mobility Model (RWPM) [10] is an adapted version of RWMM in which a pause time is inserted between the changes in direction and speed. The pause time is selected randomly from a given range. In RWPM, a destination is selected randomly from the simulation area and a node moves toward that position with a randomly chosen speed. A specific relation between the pause time and the speed can be applied based on a specific application, to fit the model towards either a more stable network or a network with frequent topology changes.

Both the RWMM and the RWPM suffer from the node concentration problem. The probability of a node being in the center of the simulation area is higher and node clusters form around the center. The Random Direction Mobility Model (RDMM) [22] tries to alleviate this problem by forcing the nodes to meet a border in each movement step. Moreover, a probabilistic version of a random walk is proposed in [7] that uses a probability matrix for determining the target position of the next step. The matrix can be set based on a specific application.

All models mentioned so far are memory-less. This means that the completed movement step does not have any impact on the decision about the movement parameters of the next step. In [15], the Random Gauss-Markov Mobility (RGMM) model is proposed in which the value of speed and direction are calculated using a combined Markovian and Gaussian distribution. By the Markovian property, the values of speed and direction at the $n^{\text {th }}$ step are calculated according to their value at the $(n-1)^{t h}$ step. On the other hand, using a Gaussian distribution with the given mean values for the direction and speed, randomness is inserted into the selection process. The impact of these two parts is controllable by setting just one tuning factor.

\subsection{Group Mobility Models}

In the wireless networks domain, there are many situations in which there are dependencies between the movement patterns of different nodes. Human mobility patterns mostly show such group behaviors (for instance, because of social activities). Accordingly, several mobility models have been proposed in the literature that each try to model the 


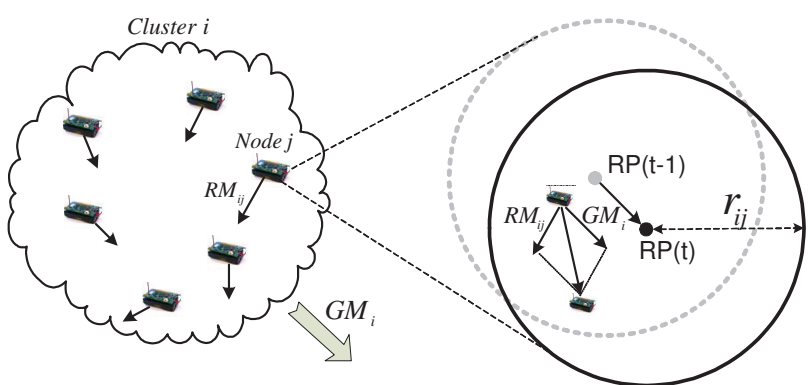

Figure 1: Node movement in the RPGM model [18]

movement pattern of humans in specific scenarios. The column mobility model, the pursue model, and the nomadic community model, all presented in [23], are some early approaches to model such correlated mobility patterns. Recent activities are mostly based on the concept of higher node density in more popular locations such as the home location. For instance, the Small World In Motion (SWIM) model [16] presents a mobility model based on the fact that humans go more often to locations near their home and to locations in which they can meet many other people. The N-Body model presented in [26] uses some real human movement traces and tries to capture social information metrics from them. The model then synthesizes that information to make output traces reproducing the heterogeneity of the input traces.

Among all group mobility models, the Reference Point Group Mobility model (RPGM) [9] is a general model that can be tuned to model many scenarios. In fact, many recent proposed models are in some way special cases of the RPGM model. As we are using this model for our mobility model, it is explained in detail. In the RPGM mobility model, a Logical Center $(L C)$ is defined for the group of nodes, the motion of which defines the entire group movement. Every group $i$ has a group motion vector $\overrightarrow{G M}_{i}$ that determines the motion of the group's logical center $\left(L C_{i}\right)$.

A Reference Point $(R P)$ is assigned to each node in the group and determines the initial position of the node on the body. The reference point of a node is a fixed point relative to the logical center of the group. Therefore, the reference point of every node within the group $i$ moves with the group motion vector $\overrightarrow{G M}_{i}$. Fig. 1 illustrates the situation for a group. Moreover, every node $j$ moves within a predefined area (a circle or sphere with radius $r_{i j}$ ) around its reference point with a random motion vector $\overrightarrow{R M}_{i j}$. So there is independent motion of individual nodes in the group while the logical center provides the whole group movement. The location of every node after each time step is defined by the sum of the group motion and the random motion vectors.

The definition of RPGM is quite general and defines the correlation between nodes in the group. Notice that the RPGM does not prescribe a mobility pattern for moving the $L C$ or for individual movement within the group. The appropriate patterns should be designed according to the requirements of the exact scenario. By selecting a proper group motion behavior, we can model human movement, while setting the random motion vectors $\left(\overrightarrow{R M}_{i j}\right)$ to define the motion of individual sensors installed on various positions on the body.
The RPGM model has been utilized in [21] to make a model of the mobility of body sensor nodes. In this model, the global movement of the $L C$ (human movement) is done using the Random Gauss-Markov Mobility model. A swarm behavior inspired model presented in [11] and [12] is utilized for the individual movement of sensor nodes on the body. In this method, if the node is close to its reference point, the model forces it away from that position. On the other hand, if the node is far from its reference point, the swarm inspired method attracts the node towards its reference point. These movements take place within the given range around the reference point of each node.

To the best of our knowledge, the model of [21] is the only mobility model for wireless body sensor networks described in detail in the literature. However, there are several limitations in this model. First, the model uses certain mobility models for moving the logical center of the group and individual node mobility. It is not clear why these models should be proper choices for a WBAN. Further, it makes the model unadaptable for different applications and sometimes the model is far from real world mobility patterns. Second, the model does not specifically implement different postures of a human body. Postures are of great importance in WBANs as the network topology may entirely change due the posture changes. Further, in a real WBAN, movement parameters of the model such as the speed and target positions of the human strongly depends on the posture. Third, the model as it has been presented only considers position and motion in two dimensions which is unrealistic for WBANs.

In this paper, we present the MoBAN mobility model for evaluating intra- and/or extra-WBAN communication with a focus on completeness, configurability, and availability of the model. It can be configured to model the mobility patterns in various application scenarios of wireless body area networks. We have used earlier versions of the model in two earlier publications, [17] and [18], to evaluate the protocols proposed in those papers. The current paper describes the model in detail, and makes it available to the scientific community.

\section{MOBILITY MODEL FOR WBANS}

A mobility model for mobile networks determines the node positions at any instance of simulation time and so influences the network topology and link properties. Consequently, the precision of the model has a major impact on the precision of the evaluated network performance. A proper mobility model for WBANs should be able to statistically model the right movement patterns of the individual nodes installed on the body as well as the whole body movement. At the same time, it should be adaptable for various application scenarios in which the movement patterns, the human activities, and the surrounding environment may differ. We first explain the general structure of our mobility model (MoBAN) and then present its constituent blocks in more detail.

\subsection{Model Structure}

The RPGM [9] model constructs the basic platform for modeling a WBAN in our model. In fact, the RPGM model determines the grouping strategy of MoBAN. We extend the RPGM model by introducing postures which have individ- 


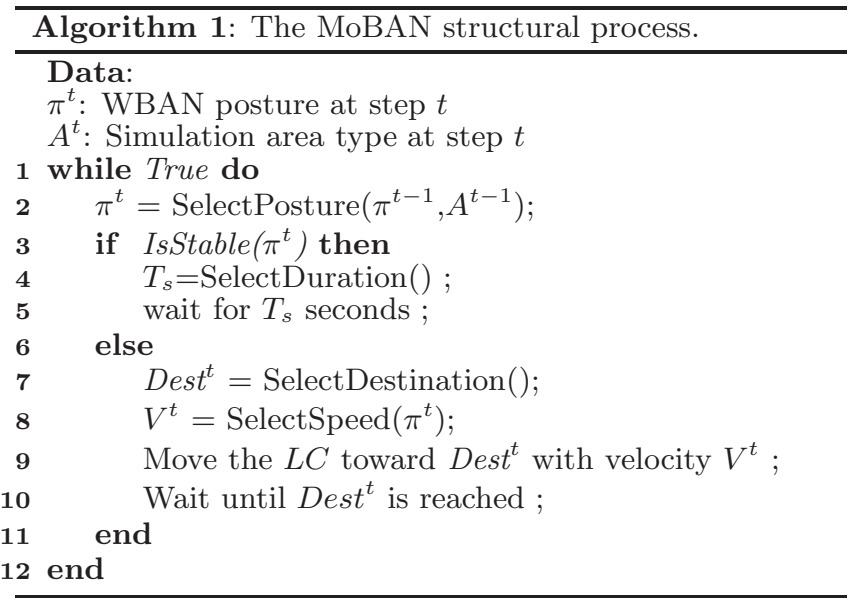

ual mobility parameters. The MoBAN itself is constructed by two basic control units which are the posture selector and the global movement module. On the one hand, the posture selector process determines the current posture at any instance of time. The individual movement (the random motion vector) of every sensor node is subsequently determined according to the selected posture. On the other hand, the global movement process is responsible for controlling the mobility of sensor nodes on the body as a whole (i.e. moving the logical center of the group). The parameters of this movement are of course not independent from the selected posture and the exact application scenario.

A mobility pattern starts with the posture selector process. Once the posture is selected for the next mobility phase, information related to the selected posture will be retrieved. If the posture has been defined as a stable posture, such as lying down, the posture selector process keeps the control and waits till the selected time duration expires. It then selects another posture based on its strategy. In the case of a mobile posture, such as walking, the global movement control module starts moving the whole WBAN taking the parameters related to that specific posture into consideration. The movement behavior, like the destination, speed, and the path to that destination, depends on the specific strategy for the running application scenario. Once the WBAN reaches the destination point, the posture selector module gets the control to select the next posture and the process is continued. Algorithm 1 presents this process. The rest of this section explains the different functions in Algorithm 1 .

\subsection{Posture Specification}

Let $S=\left\{s_{1}, s_{2}, \ldots, s_{N_{s}}\right\}$ be the set of $N_{s}$ sensor nodes in the WBAN. Moreover, in the mobility model, let there be $N_{p}$ different postures $\pi=\left\{\pi_{1}, \pi_{2}, \ldots, \pi_{N_{p}}\right\}$. Each posture is defined by a set of specification parameters which are application dependent and should be specified by the user. The specification of each posture includes relative node positions (reference points), the radius of a sphere around the reference point of any node, the velocity value of the local movement within the sphere, and the speed range of the whole body movement (global movement), using the notations in Table 1.
Table 1: The basic specification of the postures $\pi_{j}$

\begin{tabular}{|l||l|}
\hline Notation & Description $\left(1 \leq i \leq N_{s}, 1 \leq j \leq N_{p}\right)$ \\
\hline$R P_{i j}$ & Relative reference point of node $s_{i}$ \\
$V_{i j}$ & Velocity value of node $s_{i}$ \\
$r_{i j}$ & Radius of movement sphere of node $s_{i}$ \\
$V_{\text {min }_{j}}$ & Minimum velocity of the WBAN \\
$V_{\text {max }_{j}}$ & Maximum velocity of the WBAN \\
\hline
\end{tabular}

A posture can be mobile or stable, based on the speed range of its global movement $\left(V_{\max }\right)$ which will be given based on the application scenario. In general, typical mobile postures are walking and running. But in specific applications such as a hospital situation, sitting or even lying down can be thought of as mobile postures when some sort of carrier like a wheelchair or mobile bed is used. A posture can also include more detail about the physical channel characteristics as discussed later.

\subsection{Local Mobility}

Individual movement of any sensor node (say node $s_{i}$ ) within the WBAN strongly depends on the selected posture. Based on the RPGM model, every node moves within a given sphere around its reference point. The movement parameters which are the speed $\left(V_{i j}\right)$ and the radius $\left(r_{i j}\right)$ of the movement sphere around the reference point are obtained from the specifications of the selected posture $\pi_{j}$ shown in Table 1 . The values are given based on the expected movement behavior of the node deployed on that specific position in the current posture. A node on an arm can have a very high mobility in the running posture whereas the node deployed on the chest is fixed and has no local mobility (zero velocity). However, even a node on the arm has a very low mobility in the lying down posture.

We use the random walk mobility model (RWMM) [27] in $3 \mathrm{D}$ space to determine the random motion vector $\overrightarrow{R M}_{i j}$ in each local movement step. In each step, a point within the sphere space is selected uniformly randomly as the destination. The node then moves toward the destination with the given velocity value $V_{i j}$.

\subsection{Posture Selection Strategy}

Posture selection is a very important part of the model as the posture strongly influences the network topology and node connectivity. The selected posture also determines the local movement of sensor nodes and the global mobility of the whole WBAN. Therefore, it affects the connection between the nodes in the WBAN and the external network like other WBANs or the surrounding ambient sensor network. Experimental measurement results in [20] show how Packet Delivery Ratio (PDR) and link connectivity between different sensor nodes deployed on a human body vary over different postures. As the communication protocols are expected to support such changes in the network topology and link quality, the mobility model should model posture changes carefully to evaluate the protocol correctly.

A posture pattern is a sequence of selected postures in a certain period of time (for example, the whole simulation time). Statistically, in reality, some posture patterns take place with a higher probability than other patterns. For 


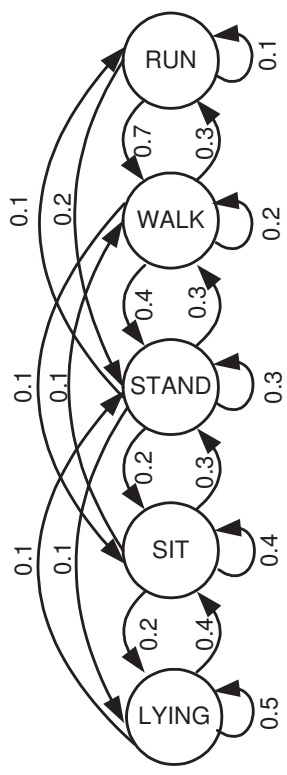

(a)

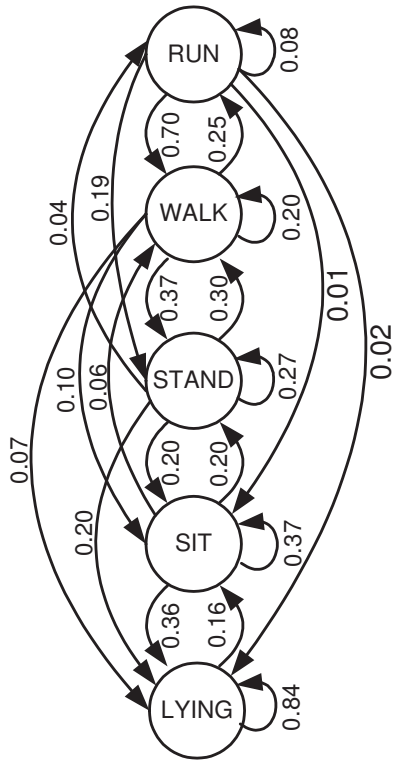

(b)
Figure 2: a) A typical Markov model for posture pattern selection used as the initial model. b) The updated Markov model based on the given steadystate vector of posture distribution $\Pi^{\mathrm{A}_{0}}$ for a certain area type $A_{0}$.

example, changing the posture from lying down to running is very rare whereas changing to the sitting posture is very likely. We use a one-level Markov model to model pattern sequences while maintaining randomness of the posture selection. We may use multiple Markov models to differentiate between area types (see below for details). Using such a Markov model, the current posture at step $t\left(\pi^{t}\right)$ is taken into consideration for selecting the next one $\left(\pi^{t+1}\right)$. The Markov model is described by a transition probability matrix $\mathrm{P}$ in which $P_{i j}$ stands for the probability of the transition from posture $\pi_{j}$ to $\pi_{i}$. In a Markov model, transitions originating from a specific state (posture) should add up to one $\left(\sum_{i=1}^{N_{p}} P_{i j}=1,1 \leq j \leq N_{p}\right)$.

The transition matrix $(\mathrm{P})$ can be obtained from real human posture traces. Fig. 2(a) shows a typical Markov chain including five different postures, namely running, walking, standing, sitting, and lying down (the figure uses abbreviated terms for the postures). The edges are labeled with the transition probabilities.

An important decision that should be made is the time duration of postures. In the case of a mobile posture, the time duration is indeed built-in as the next posture selection is done upon reaching the selected destination. For a stable posture, the posture selector process waits for a certain duration $\left(T_{s}\right)$ and then selects the next posture according to the Markov model.

As a posture change may influence the topology of the WBAN, several adaptation mechanisms have been proposed in protocols to configure and adapt the network, accordingly. Reconstructing the routing structure in [5] and trans- mit power adaptation in [17] can be mentioned as examples. These adaptations may take some time depending on the method. Therefore, having realistic posture durations is important for evaluating the applicability of a protocol. Using realistic durations, one is also able to find the right values for the parameters of the protocol to realize the expected behavior in the target application.

In our WBAN mobility model, we ask the user to specify a desired distribution for the time duration of each posture according to the application scenario. This can simply be a constant time duration or a uniform distribution, or a more precise distribution closer to real-life posture durations. The function SelectDurations() in Algorithm 1 uses the given distribution to select the posture time duration $\left(T_{s}\right)$ upon the selection of a stable posture.

\subsection{Location and Posture Pattern Coherency}

Posture selection based on a Markov model is the basic abstraction in our model. Given the Markov model, we decide about the posture at any time considering the current posture and the transition probability matrix. However, for many applications, the likelihood of posture patterns may depend on the location in the area of simulation. To take this correlation between the WBAN position and the posture pattern into account, we provide the user of the model with different abstraction levels for modeling. Let $A=\left\{A_{1}, A_{2}, \ldots, A_{N_{d}}\right\}$ be a partitioning of the simulation area into $N_{d}$ different area types. So, in any type of location, we may have different posture patterns. As an example, different rooms in a building can be thought of as having statistically different posture patterns. The posture pattern in a bedroom is surely different from the pattern in the living room or the kitchen.

For the most precise level of specification, a user can define a dedicated Markov model for any location of the area. In this case, $N_{d}$ different transition probability matrices should be given.

At a coarser level of specification, we just ask a user to specify a Markov chain with an initial transition matrix $(\mathrm{P})$ for posture changes plus the steady-state probability vector $\left(\Pi^{A_{k}}\right)$ that determines the distribution of postures in each area type. Thus $\Pi_{\mathrm{i}}^{\mathrm{A}_{\mathrm{k}}}$ stands for the steady-state probability of posture $\pi_{i}$ in area type $A_{k}$. Then the Markov model with initial transition probabilities can be automatically updated to take this probability vector into consideration. Consequently, the transition matrix $\mathrm{P}^{\mathrm{A}_{\mathrm{k}}}$ for any area type $A_{k}$ is extracted by adapting the initial transition matrix $\mathrm{P}$ to satisfy the desired steady-state vector $\Pi^{\mathrm{A}_{\mathrm{k}}}$.

So, on the one hand, the goal is to find a transition probability matrix (Markov chain $\mathrm{P}^{\mathrm{A}_{\mathrm{k}}}$ ) with the steady-state probability vector $\Pi^{A_{k}}$, which should satisfy $\mathrm{P}^{\mathrm{A}_{\mathrm{k}}} \cdot \Pi^{\mathrm{A}_{\mathrm{k}}}=$ $\Pi^{A_{k}}$. On the other hand, the resulting transition matrix should be as much as possible similar to the initial transition matrix $\mathrm{P}$. We translate this to minimizing $\left\|\mathrm{P}^{\mathrm{A}_{\mathrm{k}}}-\mathrm{P}\right\|_{F}$ where $\|\cdot\|_{F}$ stands for the Frobenius norm (which is a standard distance metric for matrices). In other words, if we let $\mathrm{D}=\mathrm{P}^{\mathrm{A}_{\mathrm{k}}}-\mathrm{P}$, the aim is to minimize $\|\mathrm{D}\|_{F}$ such that

$$
\mathrm{D} \cdot \Pi^{\mathrm{A}_{\mathrm{k}}}=\mathrm{P}^{\mathrm{A}_{\mathrm{k}}} \cdot \Pi^{\mathrm{A}_{\mathrm{k}}}-\mathrm{P} \cdot \Pi^{\mathrm{A}_{\mathrm{k}}}=\Pi^{\mathrm{A}_{\mathrm{k}}}-\mathrm{P} \cdot \Pi^{\mathrm{A}_{\mathrm{k}}}
$$

Since $\Pi^{\mathrm{A}_{\mathrm{k}}{ }^{T}}$ is the pseudo inverse of $\Pi^{\mathrm{A}_{\mathrm{k}}}$, the minimum norm 
solution for $\mathrm{D}$ will be as follows.

$$
\mathrm{D}=\left(\Pi^{\mathrm{A}_{\mathrm{k}}}-\mathrm{P} \cdot \Pi^{\mathrm{A}_{\mathrm{k}}}\right) \cdot \Pi^{\mathrm{A}_{\mathrm{k}} T}
$$

As we supposed that $\mathrm{D}=\mathrm{P}^{\mathrm{A}_{\mathrm{k}}}-\mathrm{P}$, then the solution for the new transition matrix is calculated by Eqn. 3 .

$$
\mathrm{P}^{\mathrm{A}_{\mathrm{k}}}=\mathrm{P}+\left(\mathrm{I}_{\mathrm{N}_{\mathrm{p}}}-\mathrm{P}\right) \cdot \Pi^{\mathrm{A}_{\mathrm{k}}} \cdot \Pi^{\mathrm{A}_{\mathrm{k}}^{\mathrm{T}}}
$$

where $\mathrm{I}_{\mathrm{N}_{\mathrm{p}}}$ stands for the identity matrix of size $N_{p}$.

The resulting transition matrix can be proven to be Markovian by showing that every column of the matrix $\mathrm{P}^{\mathrm{A}_{\mathrm{k}}}$ adds up to one. Note that all entries of the transition matrix should be a probability value $\left(0 \leq P_{i j}^{A_{k}} \leq 1\right)$. We take that into account for computing the matrix. To do so, it may be necessary to modify the resulting matrix and repeat Eqn. 3 in a recursive manner till we obtain a proper transition matrix satisfying the desired steady-state probabilities.

As an example, consider the initial Markov model of Fig. 2(a) with the transition matrix $\mathrm{P}$ given in Eqn. 4.

$$
\mathbf{P}=\left(\begin{array}{ccccc}
0.1 & 0.3 & 0.1 & 0 & 0 \\
0.7 & 0.2 & 0.3 & 0.1 & 0 \\
0.2 & 0.4 & 0.3 & 0.3 & 0.1 \\
0 & 0.1 & 0.2 & 0.4 & 0.4 \\
0 & 0 & 0.1 & 0.2 & 0.5
\end{array}\right)
$$

Now, suppose that we specify a steady-state posture distribution $\Pi^{\mathrm{A}_{0}}$ for the area type $A_{0}$ as in Eqn. 5. This can be thought of as a distribution for a bed room for example.

$$
\boldsymbol{\Pi}^{\mathbf{A}_{\mathbf{0}}}=\left(\begin{array}{c}
0.025 \\
0.075 \\
0.1 \\
0.2 \\
0.6
\end{array}\right)
$$

The new Markov model $\mathrm{P}^{\mathrm{A}_{0}}$ for this area type is calculated using Eqn. 3 as follows.

$$
\mathbf{P}^{\mathbf{A}_{\mathbf{0}}}=\left(\begin{array}{ccccc}
0.0812 & 0.2544 & 0.0392 & 0 & 0 \\
0.6959 & 0.1982 & 0.2976 & 0.0649 & 0 \\
0.1881 & 0.3749 & 0.2665 & 0.2027 & 0 \\
0.0140 & 0.0995 & 0.1993 & 0.3682 & 0.1644 \\
0.0208 & 0.0730 & 0.1974 & 0.3643 & 0.8357
\end{array}\right)
$$

Fig. 2(b) shows the resulting Markov chain for the area type $A_{0}$ (the probability values in the figure have been rounded). This model satisfies the given steady-state postures distribution and it still takes the more likely transition into account based on initial Markov model P. Thus, the output posture pattern will be more realistic.

As it is observed, the model can be configured to be very specific for a particular scenario by providing one Markov chain for each area type of the simulation area, or it can be more general through utilizing a less precise model specification which will be more convenient for the user.

\subsection{Global Movement}

When a mobile posture is selected, a target position should be chosen to start movement of the whole WBAN (moving the $L C$ of the group). A uniform random strategy can be applied as the basic level if the probability of being selected as the target position for all area types of the area is the same in the application. There is also the possibility to have a specific non-uniform WBAN position distribution for different area types of the simulation area. For instance, we may specify a value $p_{i}$ as the probability of the area type $A_{i}$ being selected as the target position for the movement of the WBAN.

Doing so, different WBANs in the network move independently from each other. However, in many applications, modeling the social activities (e.g., meeting in a room) is of great importance as it can change many things in the network, like interference, wave propagations, and the connectivity between WBANs and the WSN infrastructure. According to the application scenario, a community-based mobility model in the existing literature of mobile ad-hoc wireless networks (e.g. models presented in [14],[16],[23],[26]) can be chosen to be used on top of our WBAN mobility model to include social activities as well.

\subsection{Temporal Correlation}

The spatial correlation has been taken into account in the MoBAN mobility model through the possibility of specifying location-dependent distributions for the posture pattern (Markov chain), and WBAN target position selection, as described. However, in many applications in reality, time is also important. For instance, the area types that a human (WBAN) may visit during day time and night time are different. As a solution, one can independently conduct several simulation runs with different parameters and distributions to check the performance of the network in different time frames. Nevertheless, integrating such a facility in the mobility model is worthwhile and makes the model more convenient to use.

Temporal correlations can be accordingly integrated into the model by performing the space and time partitioning with the same mechanism that was already explained. It means both the target position and the posture pattern selection processes can be done taking the time into consideration as well. To do so, the simulation area is partitioned into $N_{d}$ different area types as explained in Section 3.5. Each area type then is partitioned into $N_{t}$ separate time frames. By this, we have $N_{d} \times N_{t}$ space-time partitions in which $A_{i, j}$ is set to be the $i^{\text {th }}$ spatial area type in the $j^{\text {th }}$ time frame. Now, different distributions can be specified for different space-time partitions based on the application scenario and the required level of precision.

\subsection{WBAN Radio Model Parameters}

The human body has a severe influence on radio wave propagation, and so it affects the connectivity and the topology of the WBAN. Thus it is very important to take the body effect on propagation loss and link quality in various postures into consideration. As the effect of the body strongly depends on the relative position of nodes and the body situation, it is very useful to include that in the WBAN mobility model as the mobility model is responsible for the position of nodes at any instance in time.

Based on the radio propagation model which is used for network simulation, we can decide about the channel parameters in the mobility model according to the current situation of the body. Provided that a path-loss model is used, for instance, we specify for every pair of nodes in the definition of each posture, the mean $\left(\mu_{\alpha}\right)$ and deviation $\left(\sigma_{\alpha}\right)$ of the path 


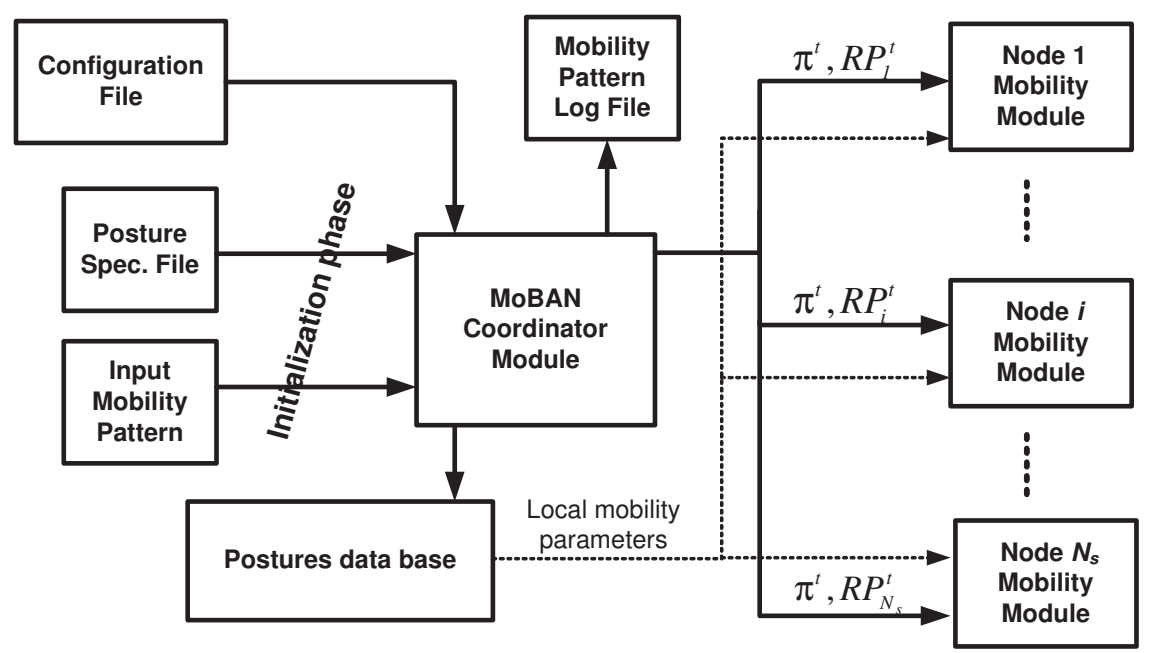

Figure 3: Block diagram of the OMNeT++ implementation of the MoBAN mobility model.

loss coefficient $\alpha$. The mean value is specified (in the range from 3 to 7 , see [25]) according to the ratio of the distance between the pair of nodes in which radio waves should be propagated around or through the body. The deviation is set based on the relative mobility of the pair of nodes in the specific posture. The real values for the mean and deviation parameters can be extracted from the result of a real experiment. During the simulation run, a value is selected for $\alpha$ according to the normal distribution $\mathcal{N}\left(\mu_{\alpha}, \sigma_{\alpha}\right)$ at the start time of every selected posture.

\section{IMPLEMENTATION ISSUES}

We implemented the MoBAN mobility model as an addon to the mobility framework of the OMNeT++ discrete event simulator, to be used for our own research as well as to make it available to the scientific community for other research on WBAN protocols. The implementations of the WBAN mobility model as well as the pure RPGM group mobility model are available through the web site http://www.es.ele.tue.nl/nes/. Any number of WBANs can be easily instantiated and different WBANs can include different numbers of sensor nodes. The ambient network or non-WBAN nodes can still be involved and can have their own mobility using existing singular node mobility models existing in the mobility framework of OMNeT++. By this, a complete combined network consisting of several WBANs and an ambient sensor network can be simulated.

\subsection{MoBAN Implementation Modules}

Figure 3 shows the block diagram of the implementation of the mobility model for a single WBAN. To have a WBAN in the network, one mobility coordinator module is instantiated in the top level simulation setup as well as one mobility module inside each node within the WBAN. In the case of multiple WBANs, multiple coordinators should be instantiated and the mobility module in each sensor node specifies the enclosing WBAN coordinator module.

The mobility coordinator is the main module that provides the group mobility and correlation between nodes in a WBAN. In the initialization phase, it reads several user de- fined files. First of all, it reads the postures specification file and makes a database of that information to be used during simulation by the coordinator module itself or by other nodes. Any node can access the posture database during simulation to retrieve information about its local mobility parameters (current posture, $V_{i j}$, and $r_{i j}$ ). A configuration file as well as all required distributions for specifying different non-uniform distributions is also read in the initialization phase. Note that all WBANs may use a single posture or configuration file if they are in the same situation. However, different input files can also be specified to have variety between different WBANs in a simulation run.

During the simulation, the mobility coordinator decides about the posture and the global movement of the whole WBAN (position of the $L C$ ) by implementing the posture selector and global movement processes of the model based on Algorithm 1. The $L C$ 's position of the WBAN is an absolute position within the (three-dimensional) simulation area and is determined according to the mobility model which is being used for movement of the whole WBAN (Section 3.6). The coordinator module also knows the reference point $(R P)$ of each node in the current posture as well, as a relative position to the $L C$. What a node $s_{i}$ within the WBAN needs is actually its absolute reference point $\left(R P_{i}^{t}\right)$ as well as the current posture $\left(\pi_{j}\right) . R P_{i}^{t}$ is calculated by adding the current position of the logical center $\left(L C^{t}\right)$ by the reference point of the node $s_{i}$ in posture $\pi_{j}\left(R P_{i}^{t}=L C^{t}+R P_{i j}\right)$. The mobility coordinator sends the new $R P_{i}^{t}$ to every node belonging to the WBAN.

The mobility module of a sensor node $s_{i}$ receives the $R P_{i}^{t}$ and the current posture $\pi_{j}$ of the WBAN from its coordinator module. Subsequently, it retrieves the parameters of the local movement $\left(V_{i j}, r_{i j}\right)$ from the posture database. It decides about the relative position $\left(L_{i}^{t}\right)$ of the node relative to the $R P_{i}^{t}$. Finally, it sets $L_{i}^{t}+R P_{i}^{t}$ as the physical position of the node $s_{i}$ at that time instance. Note that the individual node mobility module has nothing to do with a posture change as its reference point is properly updated upon a posture change by the coordinator module. It only retrieves the local movement parameters of the new posture. 


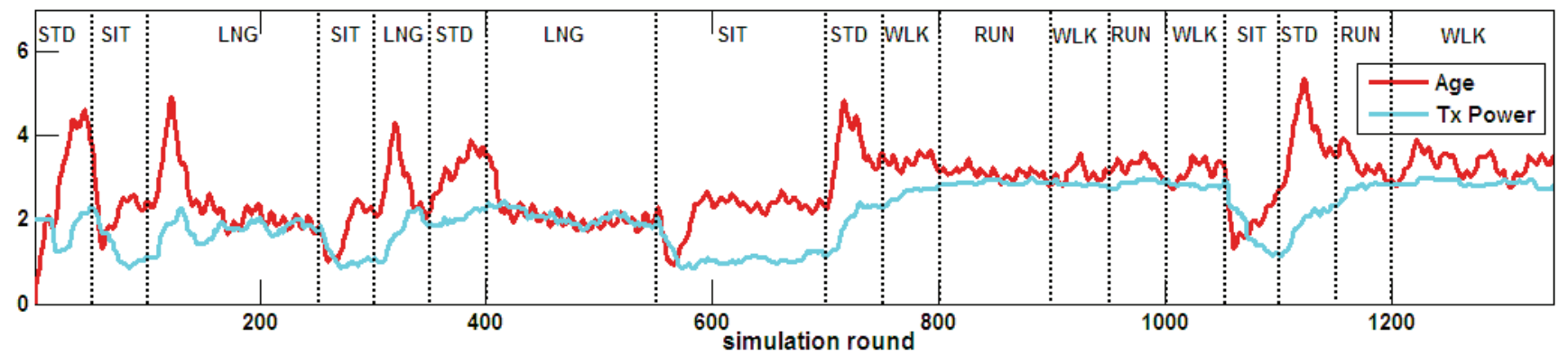

(a) Average age of data items at the gateway and average transmit power of a node in simulation using MoBAN

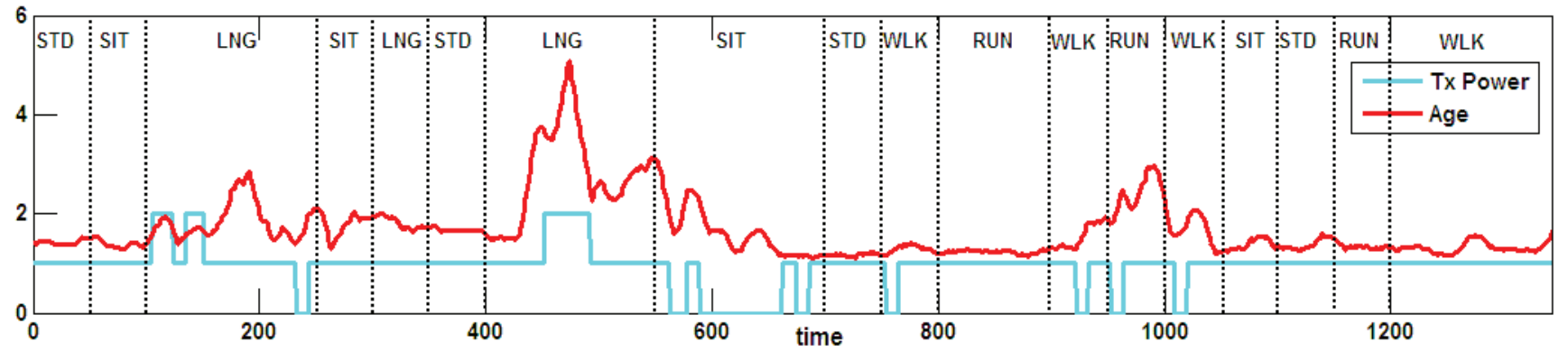

(b) Average age and Tx power level of a node in a real experiment

Figure 4: Simulation results for an intra-WBAN communication protocol obtained using MoBAN and results of a real experiment.

\subsection{Recording and Reusing Mobility Patterns}

The mobility pattern influences the performance evaluation results of a network protocol when there is some form of mobility in the network. This effect is more important in simulating networks including WBANs because of the high mobility in these networks. On the other hand, sometimes we simulate a protocol to compare its performance with alternative protocols. In such a situation, to have fairer comparison, it is worthwhile to use the exact same mobility pattern for simulating different protocols.

In our implementation of the WBAN mobility model, we have the feature of logging the mobility pattern of a WBAN. The coordinator module logs the selected destinations, velocity values and posture pattern in a file if the logging function is requested. The coordinator module can be set to read a previously logged mobility pattern of an earlier simulation run by specifying the name of the input mobility pattern file. To simulate different protocols for a given network, we may run the first simulation and log the mobility pattern. Then, for the rest, we use the logged pattern.

\section{CASE STUDIES}

We used MoBAN with special configurations to evaluate communication protocols that we have presented in our earlier publications [17] and [18]. In this section, the impact of the mobility model on simulations is investigated. Moreover, as these two case studies concern different protocols (one for intra- and another one for extra-WBAN communication), the applicability and configurability of the model for different applications are illustrated.

\subsection{Intra-WBAN Protocol Stack}

In [17], we proposed a multi-hop protocol stack for intraWBAN communication aiming to provide robustness against network topology changes due to the posture changes and low link quality in WBANs. All sensor nodes need to send their data items to a gateway node on the body. Because of a very short transmission range of wireless devices deployed on the body, nodes may not be able to reach the gateway in one hop. The protocol suggests a gossip-based [8] routing mechanism together with an appropriate TDMA-based Medium Access Control (MAC) to reliably deliver information to the gateway node through one or more hops. As the protocol does not rely on any specific routing structure like a tree, there is no need for reconstructing the routing structure upon network topology changes (due to the posture changes and node mobility). This makes the protocol robust against those changes in the network topology. Moreover, a transmit power adaptation mechanism is proposed to optimize the transmit power consumption of sensor nodes while realizing the proper node connectivity to meet the quality-of-service requirements.

To evaluate the performance and robustness of such a protocol, a WBAN mobility model including different postures is the first prerequisite as the protocol aims to provide robustness with respect to such topology changes. We used a particular configuration of MoBAN to this aim. Twelve sensor nodes including the gateway node are supposed to be installed on different positions in the WBAN. Accordingly, the specifications of five different postures are defined and the initial Markov model of Fig. 2(a) is used to generate the 
posture pattern. Since the protocol is designed for intraWBAN use, just one WBAN instance is sufficient as the simulation setup. Since there are random processes in the communication protocol, to have statistically more reliable results, the simulation was run for 32 different seeds for the random generator. We used the mobility pattern recording and reusing capability of the model implementation to have the same mobility patterns for conducting simulations using different seeds.

Fig. 4(a) shows a part of the simulation result in which the average age of data items at the gateway node is shown. The age for data from a node at the gateway at any point in time is defined as the time difference between the current time and the sample time of the last received data item of that node within the WBAN. The sensitivity of the protocol in terms of the average age as well as the reaction of the protocol through changing the transmit power are visible in the figure. It is best visible in changing from closed postures (e.g., sitting) to open postures (e.g., standing) and vice versa. Abrupt changes in the value of the average age reveal the impact of different postures on the connectivity of the WBAN nodes. Transmit power adaptation tries to keep the age level within a desired range. The figure also includes the transmit power trend of a node deployed on the feet to show how the posture changes affect the settings of a node. The transmit power is selected among four levels, denoted $0,1,2$, and 3 . The graph shows the average over the 32 runs.

To confirm that network connectivity and quality of services depend on the posture, we conducted a real experiment using nine MyriaNed [24] sensor nodes deployed on a human body. The experiment was done with the same posture pattern as the simulations. Nevertheless, the experimental setup differs from the simulation setup in various aspects (e.g., the number of nodes, radio behavior, and individual mobility of nodes). Thus, the results are not comparable with the simulations. However, the experimental curve in Fig. 4(b) shows an instance of the effect of posture changes on the quality of service, the network performance, and the setting of a node (transmit power level, 0, 1, 2, or 3) in real world WBANs.

The experimental results show age peaks and transmit power variations as are also seen in the simulation experiments. This provides an indication that MoBAN captures posture changes as required for simulations, although more experiments are needed with mobility traces from real applications. Such traces are not yet available for WBAN-based applications. Traces available for ad-hoc networking [3] are not suitable for evaluating the WBAN mobility patterns.

\subsection{Extra-WBAN Communication Protocol}

As the second case study, we show the usability of the model for performance evaluation of an extra-WBAN communication protocol. In the presence of an ambient network, WBANs behave like clusters of mobile nodes within that network. A Medium Access Control protocol is designed in [18] to support cluster (WBAN) mobility in large-scale wireless sensor networks. The protocol is based on the TDMA strategy for accessing the shared medium. Cluster mobility is supported by dedicating a specific part of the TDMA frames to the sensor nodes within the mobile clusters. Moreover,

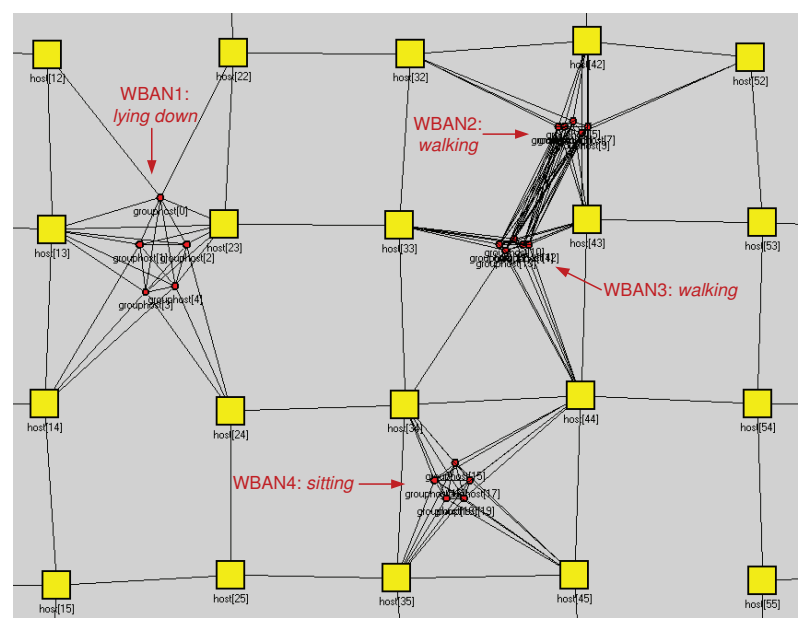

Figure 5: A snapshot of the simulation for extraWBAN communication using MoBAN; view from above.

a CSMA-based mechanism has been used for accessing this part of the frames by sensor nodes from different clusters.

For performance evaluation of the protocol, we made a simulation setup including 100 ambient (fixed) sensor nodes placed around fixed grid points as well as up to four WBANs each including five sensor nodes. WBANs move independently within the simulation area. Fig. 5 depicts what the simulation looks like in OMNET ++ at a particular time instance. The lines in the figure show potential wireless links between sensor nodes. Four WBANs as well as their current postures are shown. WBAN2 and WBAN3 are in the potential interference range of each other. So they may interfere or hear each other. This affects the performance of the system and is quite important in the investigation of the behavior of the proposed protocol in such situations. Simulation results presented in [18] show that the inter-WBAN collision ratio grows with increasing the number of WBANs in the simulation area. The collision ratio definitely depends on the time durations that different WBANs are close together. This means that modeling the WBAN movement according to the target application is of great importance for decisions about the applicability of the protocol.

\section{CONCLUSION}

This paper presents MoBAN, a Mobility model for wireless Body Area Networks. The model has been specifically designed so that it can be configured for being used for performance evaluation of a broad range of application scenarios including WBANs. Both global movement of the WBAN and the individual node mobility within the WBAN have been taken into consideration. The model can be used in simulating both intra- and extra-WBAN protocols. Two case studies are shown that use the specific configurations of the model for different protocols. The implementation of the model as an add-on to the mobility framework of the OM$\mathrm{NeT}++$ event simulator makes the model available to the scientific community to be used for research on WBAN communication protocols and applications. The implementation of MoBAN as well as the RPGM group mobility model on 
top of the OMNeT ++ simulator can be obtained through http://www.es.ele.tue.nl/nes/.

As future work, we plan to perform additional experiments with real WBAN deployments to validate and tune MoBAN. Other researchers are invited to do so as well. Currently, no real life WBAN mobility traces for realistic application scenarios are available for research purposes. With real traces, like those available for ad-hoc networking [3], it would be possible to further test and tune the MoBAN mobility model.

\section{REFERENCES}

[1] Castalia simulator website. http://castalia.npc.nicta.com.au.

[2] OMNeT++ Network Simulator website. http://www.omnetpp.org.

[3] SUMATRA: Stanford University Mobile Activity TRAces. http://infolab.stanford.edu/pleiades/sumatra.html.

[4] The Network Simulator-NS2/3 website. http://www.isi.edu/nsnam/ns/.

[5] B. Braem et al. The wireless autonomous spanning tree protocol for multihop wireless body area networks. In Proc. 3rd Int'l Conf. on Mobile and Ubiquitous Systems (MobiQuitous), pages 284-291. IEEE, 2006.

[6] T. Camp, J. Boleng, and V. Davies. A survey of mobility models for ad hoc network research. Wireless Communications and Mobile Computing (WCMC): Special Issue on Mobile Ad Hoc Networking: Research, Trends and Applications, 2(5):483-502, 2002.

[7] C. Chiang. Wireless Network Multicasting. PhD thesis, University of California, Los Angeles, 1998.

[8] D. Gavidia and M. van Steen. A probabilistic replication and storage scheme for large wireless networks of small devices. In Proc. 5th IEEE Int'l Conf. Mobile and Ad Hoc Sensor Systems (MASS). IEEE, 2008.

[9] X. Hong, M. Gerla, G. Pei, and C. Chiang. A group mobility model for ad hoc wireless networks. In Proc. 2nd ACM Int'l Conf. on Modeling, analysis and Simulation of Wireless and Mobile systems (MSWiM), pages 53-60. ACM, 1999.

[10] E. Hyytiä and J. Virtamo. Random waypoint mobility model in cellular networks. Wireless Networks, 13(2):177-188, 2007.

[11] B. Kadrovach and G. Lamont. Design and analysis of swarm-based sensor systems. In Proc. 44th IEEE Midwest Symposium on Circuits and Systems (MWSCAS), pages 487-490. IEEE, 2001.

[12] B. A. Kadrovach and G. B. Lamont. A particle swarm model for swarm-based networked sensor systems. In Proc. ACM Symposium on Applied Computing, pages 918-924. ACM, 2002.

[13] A. Köpke et al. Simulating wireless and mobile networks in OMNeT++ - the MiXiM vision. In Proc. Conf. on Simulation Tools and Techniques (SIMUTools). ICST, Brussels, 2008.

[14] L. Kyunghan, H. Seongik, J. Seong, R. Injong, and C. Song. SLAW: A new mobility model for human walks. In Proc. 28th IEEE Conference on Computer Communications (INFOCOM), pages 855-863. IEEE, 2009.

[15] B. Liang and Z. Haas. Predictive distance-based mobility management for PCS networks. In Proc. 8th annual joint Conf. of the IEEE Computer and Communications Societies (INFOCOM), pages 1377-1384. IEEE, 1999.

[16] A. Mei and J. Stefa. SWIM: A simple model to generate small mobile worlds. In Proc. 28th IEEE Conference on Computer Communications (INFOCOM), pages 855-863. IEEE, 2009.

[17] M. Nabi, T. Basten, M. Geilen, M. Blagojevic, and T. Hendriks. A robust protocol stack for multi-hop wireless body area networks with transmit power adaptation. In Proc. 5th Int'l Conf. on Body Area Networks (BodyNets). ICST, 2010.

[18] M. Nabi, M. Blagojevic, M. Geilen, T. Basten, and T. Hendriks. MCMAC: An optimized medium access control protocol for mobile clusters in wireless sensor networks. In Proc. 7th annual IEEE Communications Society Conf. on Sensor, Mesh and Ad Hoc Communications and Networks (SECON), pages 28-36. IEEE, 2010.

[19] D. Pediaditakis, Y. Tselishchev, and A. Boulis. Performance and scalability evaluation of the Castalia wireless sensor network simulator. In Proc. Conf. on Simulation Tools and Techniques (SIMUTools). ICST, Brussels, 2010.

[20] M. Quwaider and S. Biswas. Probabilistic routing in on-body sensor networks with postural disconnections. In Proc. 7th ACM MobiWAC, pages 149-158. ACM, 2009.

[21] H. Ren and M. Meng. Understanding the mobility model of wireless body sensor networks. In Proc. IEEE Int'l Conf. on Information Acquisition, pages 306-310. IEEE, 2006.

[22] E. Royer, P. Melliar-Smith, and L. Moser. An analysis of the optimum node density for ad hoc mobile networks. In Proc. IEEE Int'l Conf. on Communications (ICC), pages 857-861. IEEE, 2001.

[23] M. Sanchez and P. Manzoni. ANEJOS: a java based simulator for ad hoc networks. Future Generation Computer Systems, 17(5):573-583, 2001.

[24] F. van der Wateren. The art of developing WSN applications with MyriaNed. Tech. report, Chess Company, the Netherlands, 2008.

[25] T. Zasowski et al. UWB for noninvasive wireless body area networks: Channel measurements and results. In Proc. Conf. on Ultra Wideband Systems and Technologies (UWBST), pages 285-289. IEEE, 2003.

[26] C. Zhao and M. L. Sichitiu. N-Body: Social based mobility model for wireless ad hoc network research. In Proc. 7th annual IEEE Communications Society Conf. on Sensor, Mesh and Ad Hoc Communications and Networks (SECON), pages 1-9. IEEE, 2010.

[27] M. Zonoozi and P. Dassanayake. User mobility modeling and characterization of mobility patterns. IEEE Journal on Selected Areas in Communications, 15(7):1239-1252, 1977. 